\title{
Bimodal biophotonic imaging of the structure-function relationship in cardiac tissue
}

William J. Hucker

Crystal M. Ripplinger

Washington University

Department of Biomedical Engineering

St. Louis, Missouri 63130

\section{Christine P. Fleming}

Case Western Reserve University

Department of Biomedical Engineering

Cleveland, Ohio 44106

Vadim V. Fedorov

Washington University

Department of Biomedical Engineering

St. Louis, Missouri 63130

\section{Andrew M. Rollins}

Case Western Reserve University Department of Biomedical Engineering Cleveland, Ohio 44106

\section{Igor R. Efimov}

Washington University

Department of Biomedical Engineering

St. Louis, Missouri 63130

\begin{abstract}
The development of systems physiology is hampered by the limited ability to relate tissue structure and function in intact organs in vivo or in vitro. Here, we show the application of a bimodal biophotonic imaging approach that employs optical coherence tomography and fluorescent imaging to investigate the structure-function relationship at the tissue level in the heart. Reconstruction of cardiac excitation and structure was limited by the depth penetration of bimodal imaging to $\sim 2 \mathrm{~mm}$ in atrial tissue, and $\sim 1 \mathrm{~mm}$ in ventricular myocardium. The subcellular resolution of optical coherence tomography clearly demonstrated that microscopic fiber orientation governs the pattern of wave propagation in functionally characterized rabbit sinoatrial and atrioventricular nodal preparations and revealed structural heterogeneities contributing to ventricular arrhythmias. The combination of this bimodal biophotonic imaging approach with histology and/or immunohistochemistry can span multiple scales of resolution for the investigation of the molecular and structural determinants of intact tissue physiology. @ 2008 Society of Photo-Optical Instrumentation Engineers. [DOI: 10.1117/1.2975826]
\end{abstract}

Keywords: biomedical optics; image processing; coherence; tomography; atrioventricular node; sinoatrial node.

Paper 08014R received Jan. 16, 2008; revised manuscript received Apr. 21, 2008; accepted for publication Apr. 22, 2008; published online Sep. 10, 2008.

\section{Introduction}

The advent of high resolution structural and functional imaging of biological tissues has created the possibility of combining imaging techniques in a novel way to probe the relationship between structure and function in many tissues. Optical coherence tomography (OCT) is a rapidly developing noncontact imaging modality that can generate three-dimensional (3D) images in real time with micron-scale spatial resolution. ${ }^{1,2}$ Optical coherence tomography is similar to ultrasound; however, instead of generating time-gated images based on echoes of sound waves, OCT images backscattered light with gating based on the interference of light coming from the tissue with light in a reference path ${ }^{3}$ and is capable of imaging a depth of 1 to $2 \mathrm{~mm}$ in cardiac tissue. ${ }^{4}$

The spatial resolution of OCT is anywhere from 1 to $10 \mu \mathrm{m}$, and it can provide very detailed morphological information in intact tissues and organs. Historically, this level of detail has been limited to histology; however, as a nondestructive imaging modality, OCT produces images of tissue structures that are free from the artifacts induced by histological sectioning. Therefore, OCT bridges the gap between the resolution of nondestructive imaging techniques, such as ultrasound and magnetic resonance imaging, and his-

Address all correspondence to Igor R. Efimov, Washington University, Department of Biomedical Engineering, 1 Brookings Dr., Campus Box 1097, St. Louis, MO 63130. Tel: 314-935-8612; Fax: 314-935-8377; E-mail: igor@wustl.edu. tology or confocal microscopy performed on tissue sections. ${ }^{5}$ Because of its resolution and real-time imaging, the applications of OCT have expanded across many fields of medicine, such as ophthalmology, ${ }^{6}$ nephrology, ${ }^{7}$ and cardiology. ${ }^{8}$

To date, the clinical applications for OCT in cardiology relate to coronary artery imaging. However in cardiac research, OCT has also been used to study the mechanical function of the embryonic mouse heart, ${ }^{9}$ and the growth mechanics of the developing chick heart, ${ }^{10}$ demonstrating that OCT can be used to investigate the relationship between cardiac structure and mechanical function. Also, two previous preliminary studies have shown that OCT can be used to recognize components of the cardiac conduction system, ${ }^{4,11}$ which demonstrates that OCT can visualize distinct anatomical structures within the myocardium.

Optical mapping of voltage-sensitive dyes is widely used to investigate the electrophysiological function of cardiac tissue. $^{12}$ The fluorescent signal that is recorded with optical mapping represents the summed electrical activity from a volume of tissue, with a depth of penetration of 1 to $2 \mathrm{~mm}^{13,14}$ However, discerning components of optical signals originating in tissue layers below the surface is difficult, particularly in structurally heterogeneous regions such as the cardiac conduction system. Detailed knowledge of cardiac structure is also important in optical mapping of arrhythmias because

$1083-3668 / 2008 / 13(5) / 054012 / 7 / \$ 25.00$ ○ 2008 SPIE 
structural heterogeneities influence arrhythmia induction, stabilization, and termination. ${ }^{15,16}$ Therefore, developing a method to correlate the 3D structure of cardiac tissue with functional mapping data is necessary, and in this study, we coupled optical imaging of action potentials with OCT to provide direct structural-functional correlations in several cardiac preparations. Our data indicate that the detailed structural imaging of OCT significantly enhances the interpretation of optical data.

\section{Methods}

\subsection{Animal Studies}

All animal studies were approved by the Institutional Animal Care and Use Committee of Washington University. New Zealand white rabbits $(n=18,2.5$ to 3 mos. old, 2 to $3 \mathrm{~kg}$ ) were anesthetized with $100 \mathrm{mg} / \mathrm{kg}$ of pentobarbital and 1000 intravenous units of heparin intravenously, after which a midsternal thoracotomy was performed and the heart removed. The heart was Langendorff perfused with oxygenated $\left(95 \% \mathrm{O}_{2}, 5 \% \mathrm{CO}_{2}\right)$ Tyrode's solution at $37{ }^{\circ} \mathrm{C}$ and received $50 \mu \mathrm{L}$ of $5-\mu \mathrm{M}$ di-4-ANEPPS (Molecular Probes, Eugene, Oregon) over $5 \mathrm{~min}$. The heart was dissected in cold Tyrode's solution $\left(0{ }^{\circ} \mathrm{C}\right)$ to create a preparation containing the atrioventricular $(\mathrm{AV})$ junction, sinoatrial node, or the right ventricular free wall. Each type of preparation was pinned to silicone with the endocardium as flat as possible. The preparations were superfused at $50 \mathrm{~mL} / \mathrm{min}$ with perfusate containing $15 \mathrm{mM}$ of the excitation-contraction uncoupler 2,3butanedione monoxime (Sigma, St. Louis, Missouri) to prevent motion artifacts.

\subsection{Optical Mapping}

A $16 \times 16$ photodiode array (C4675, Hamamatsu, Hamamatsu City, Japan) was used with an optical mapping system that has been described previously. ${ }^{17}$ Briefly, a ring of light emitting diodes (LED ring) with a wavelength centered at $520 \mathrm{~nm}$ illuminated the tissue to excite di-4-ANEPPS. Fluorescent emission passed through a 50-mm lens (1:1.4, AF Nikkor, Nikon, Tokyo, Japan) and a dichroic mirror, was long pass filtered at $610 \mathrm{~nm}$, and focused on the photodiode array. Optical signals were amplified (Innovative Technologies Network, Gaithersburg, Maryland), sampled at $1.5 \mathrm{kHz}$ (PCI 6031E, National Instruments, Austin, Texas), and then lowpass filtered at $120 \mathrm{~Hz}$. The focusing lens could be adjusted to change the spatial resolution from 0.56 to $2 \mathrm{~mm}$ per photodiode, depending on the preparation. Preparations were digitally photographed and optical mapping fields of view were marked with stainless steel pins in the tissue. Activation times were defined as the time of the maximum first derivative of the fluorescent $(F)$ signal, $d F / d t_{\max }$, and activation maps were constructed from the activation times of each photodiode. Conduction velocity vector fields were calculated from activation maps using a polynomial fitting method developed by Bayly et al. ${ }^{18}$

\subsection{Tissue Preparation}

Following optical mapping experiments, the tissue and silicone disk were placed in $3.7 \%$ formaldehyde diluted in phosphate buffered saline (Sigma-Aldrich, St. Louis, Missouri)

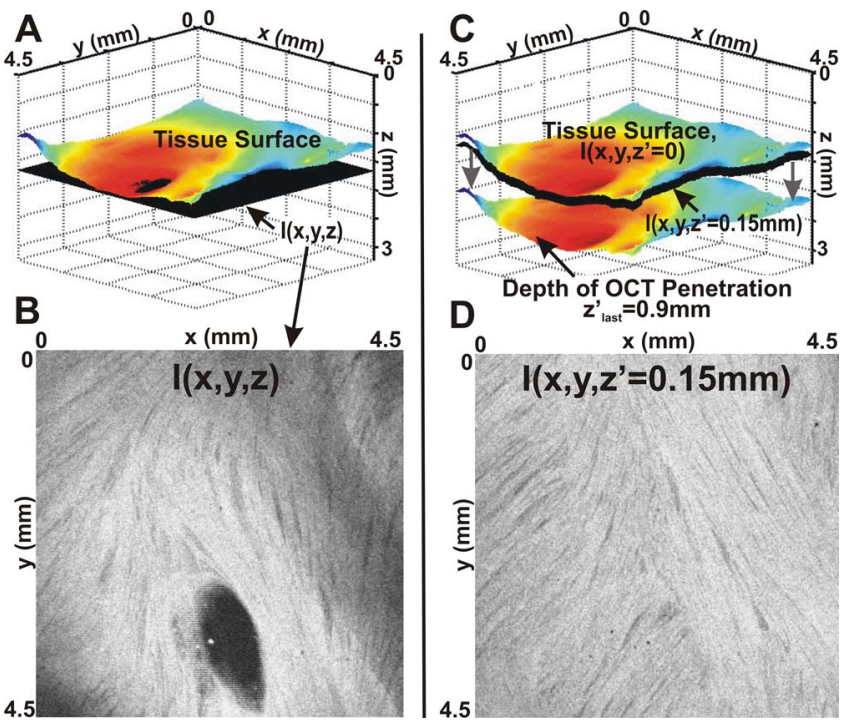

Fig. 1 (a) The surface of a representative OCT data set. Shading represent the $z$ coordinate of the surface. As shown in panel (b), sectioning the OCT data in the $z$ dimension creates an image with holes due to plane of section and shadows due to the variable depth of the section from the surface. (c) A new coordinate system $z^{\prime}$ was defined with $z^{\prime}=0$ corresponding to the tissue surface. Sectioning the tissue in $z^{\prime}$ created images that were free from artifacts due to plane of section, as seen in panel (d), an image $0.15 \mathrm{~mm}$ below the surface. This data set was sectioned in $z^{\prime}$ until fibers were no longer recognizable, which occurred at a depth of $z^{\prime}=0.9 \mathrm{~mm}$.

overnight at $4{ }^{\circ} \mathrm{C}$. The following day, samples were transferred to a $20 \%$ sucrose solution and kept at $4{ }^{\circ} \mathrm{C}$ for two days to dehydrate the tissue. Optical coherence tomography imaging was then performed with samples submerged in sucrose solution at room temperature on the second day.

\section{$2.4 O C T$}

A microscope-coupled OCT system was used to image the samples. ${ }^{19}$ A 10-mW super luminescent diode 1310-nm light source (Covega SLD 1021, Jessup, Maryland) with 70-nm resolution was used. The axial and lateral resolution of the system was approximately $10 \mu \mathrm{m}$ (in air). Individual OCT images were averaged 3 to 6 times depending on the tissue sample at the time of acquisition.

\subsection{Image Processing}

The OCT volumes were acquired as data sets that were 400 $\times 400 \times 650$ pixels in the $x, y$, and $z$ dimensions, respectively, corresponding to a tissue volume of $4.5 \times 4.5$ $\times 3.2 \mathrm{~mm}^{3}$. For each of the preparations imaged with OCT, the tissue surface was never completely horizontal across the entire imaging field of view. Depending on the tissue, surface variations ranged vertically from 0.5 to $1.5 \mathrm{~mm}$ across the $4.5 \times 4.5-\mathrm{mm}^{2}$ horizontal field of view [Fig. 1(a)]. Because of the surface variation, slicing the $3 \mathrm{D}$ data set in the $z$-plane created artifacts in the resulting images such as shadows and holes, as shown in Fig. 1(b). To generate OCT images of the tissue parallel to the surface, we defined a new coordinate system in the $z$ dimension $\left(z^{\prime}\right)$, where $z^{\prime}=0$ corresponded to the tissue surface [Fig. 1(c)]. 
To define the tissue surface, each $x z$ scan was filtered with a two-dimensional Weiner filter (MATLAB, The Math Works, Natick, Massachusetts) to remove noise before the $x z$ scans were combined to generate a $3 \mathrm{D}$ data set. Next, an intensity threshold was defined, and each column of the 3D data set was searched in the $z$ dimension for the pixel where the threshold was crossed. This point in each column corresponded to the tissue surface. All surface points were combined in three dimensions to generate the surface of the tissue. Once the surface was defined, images parallel to the surface were created by stepping through the data set below the surface by one pixel at a time in the $z^{\prime}$ dimension [Fig. 1(d)].

\subsection{Fiber Orientation Determination}

To calculate fiber orientation from two-dimensional (2D) OCT images, ${ }^{20}$ a modification of an algorithm described in Karlon et al. ${ }^{21}$ was used. First, preprocessing of the data is necessary because of the low contrast nature of OCT images. A Wiener filter was used to remove noise and a 2D Butterworth high pass filter was used to remove the low frequency background intensity (MATLAB).

The $x$ and $y$ intensity gradients $\left(G_{x}\right.$ and $G_{y}$, respectively) were computed by convolving the horizontal and vertical Sobel filters with the $2 \mathrm{D} z^{\prime}$ OCT images. For each pixel, the magnitude and angle of the gradient, $G(i, j)$ and $\theta^{\prime}(i, j)$, were calculated

$$
\begin{gathered}
G(i, j)=\sqrt{G_{x}^{2}(i, j)+G_{y}^{2}(i, j)}, \\
\theta^{\prime}(i, j)=a \tan \left(G_{y} / G_{x}\right) .
\end{gathered}
$$

Within a small subspace of the image, $W$, the dominant local direction of the fibers was computed

$$
A_{\theta}^{W}=\sum_{(i, j) W} G(i, j) \frac{\exp \left[2 \cos \left(\theta-\theta^{\prime}(i, j)\right]\right.}{\exp (2)},
$$

where $0 \mathrm{deg} \leqslant \theta \leqslant 180 \mathrm{deg}$. Vectors that were outliers were removed manually. Because the angle of the fibers is perpendicular to the angle of the gradients, the final dominant angle for the subspace of the image $W$ was determined as the maximum of the distribution $A_{\theta}^{W}$ shifted by $90 \mathrm{deg}$.

\subsection{Histology}

Following OCT imaging, the tissue was placed in optimal cutting temperature medium (Thermo Fisher Scientific, Waltham, Massachusetts), frozen, and kept at $-80{ }^{\circ} \mathrm{C}$ until use. Tissue was sectioned into $16-\mu \mathrm{m}$ sections and stained with Masson trichrome.

\section{Results}

\subsection{OCT of Myocardium}

The optical properties of cardiac fibrous tissue and fat create optical contrast within the myocardium in OCT images. Figure 2(a) illustrates the rabbit atrioventricular junction (AVJ), and corresponding OCT volumes are shown in Fig. 2(b). Comparing corresponding histology and OCT images in Figs. 2(c) and 2(d), and Figs. 2(e) and 2(f), it is clear that the fibrous tissue (which appears blue in Masson's trichrome his-

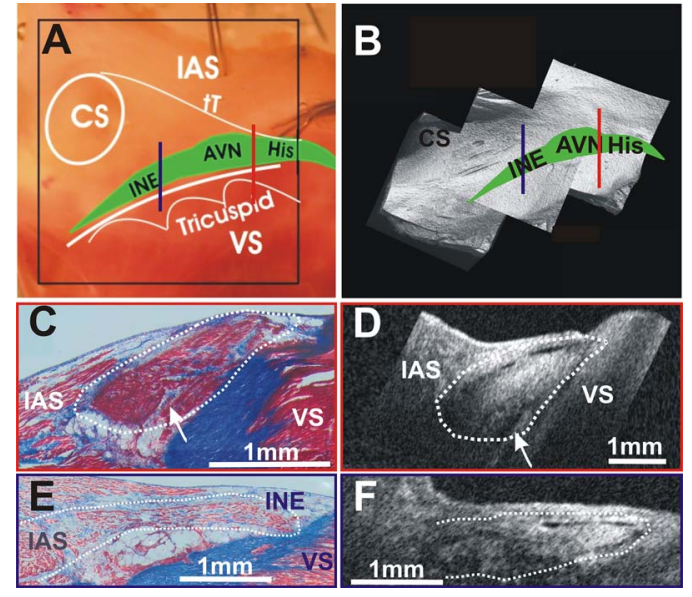

Fig. 2 (a) Preparation of the rabbit AVJ is shown, with the anatomic landmarks that surround the AV conduction system, and the location and components of the conduction system are shown in green. (b) Surface images of three OCT data sets taken from the AVJ. (c, d) Masson trichrome and the corresponding OCT image of the conduction system. Arrows denote areas of fibrous tissue within the conduction system that can be seen in the OCT image. (e, f) Masson trichrome and the corresponding OCT image of the conduction system at a different location. AVN: atrioventricular node; CS: coronary sinus; IAS: interatrial septum; INE: inferior nodal extension; His: bundle of His; tT: tendon of Todaro; Tricuspid: tricuspid valve; VS: ventricular septum. (Color online only.)

tology) and adipose tissue surrounding the conduction system in the rabbit AVJ produce less backscatter in OCT images than the myocardium, providing the necessary contrast to visualize the AVJ conduction system with OCT, free of sectioning artifacts usually present in histology.

\subsection{Optical Mapping Coupled with OCT}

Cardiac conduction is faster in the direction of fiber orientation than transverse to fibers, due to factors such as cell orientation, size, geometry, and gap junctional distribution. ${ }^{22}$ Therefore, by coupling optical mapping with OCT, we expected to find a strong correlation between conduction velocity and fiber orientation. To validate the coupling of OCT and optical mapping, we initially used a 2D system so that optical signals originating below the surface of the tissue did not confound our interpretation of the data. For this purpose, we used the rabbit sinoatrial node (SAN, $n=5)$ because the rabbit SAN is 200 to $300 \mu \mathrm{m}$ thick and several centimeters wide ${ }^{23}$ and, therefore, is essentially two dimensional with one layer of tissue contributing to optical signals [Fig. 3(a)]. Figure 3(b) displays an activation map recorded during spontaneous SAN activity. Activation began in the middle of the SAN and spread anisotropically, which is indicated by conduction velocity vectors. Velocity vectors were binned in 20-deg intervals in Fig. 3(c), which indicated that conduction spread most rapidly at $19 \mathrm{~cm} / \mathrm{s}$ at an angle of $130 \mathrm{deg}$. Conduction was slowest orthogonally at $3 \mathrm{~cm} / \mathrm{s}$.

A $z^{\prime}$ OCT image of the SAN $100 \mu \mathrm{m}$ below the surface is shown in Fig. 3(d) with the leading pacemaker location indicated, and fiber orientation of the tissue is shown in Fig. 3(e). A histogram of the fiber vectors is shown in Fig. 3(c), which indicates that the dominant fiber angle in the tissue is 


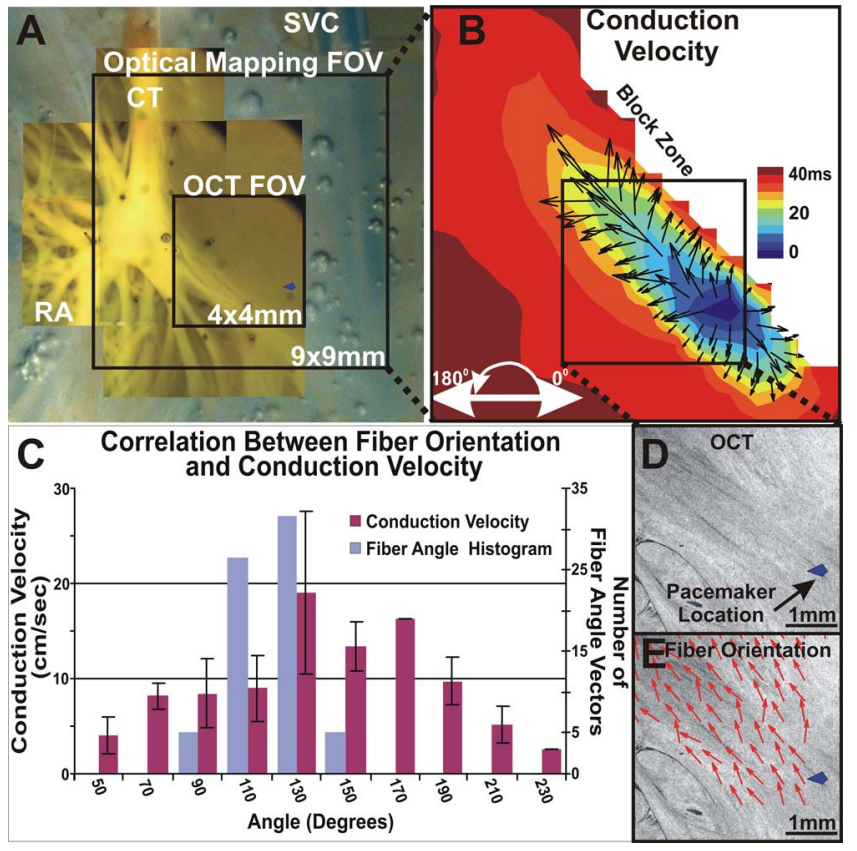

Fig. 3 (a) Rabbit sinoatrial node (SAN) preparation, with the crista terminalis (CT), right atrial free wall (RA), and superior vena cava (SVC) marked. The optical mapping and OCT fields of view (FOV) are indicated. Small blue diamond marks location of leading pacemaker. (b) Activation map of pacemaking activity in the SAN as determined with optical mapping. Conduction spread anistropically in an elliptical pattern. The block zone denotes an inexcitable region of tissue. Vectors indicate direction and magnitude of conduction velocity. (c) Plot of conduction velocity as a function of angle, as well as a histogram of the fiber orientation vectors shown in panel (f). (e) An OCT image taken $100 \mu \mathrm{m}$ below the surface. (f) Same image with fiber orientation vectors superimposed. No fiber vectors were analyzed in the trabeculated section of the image because of the artifacts induced by trabeculae with our method of sectioning. (Color online only.)

130 deg. Therefore, the direction of maximal conduction velocity was aligned with the fiber orientation of the tissue. We found the same result in all studied SAN preparations ( $n$ $=5)$.

\subsection{OCT Can Resolve Multilayer Conduction Seen in Optical Mapping}

As mentioned previously, optical mapping data consists of fluorescence that originates not only in the tissue surface, but also from tissue layers below the surface. In tissue with multiple layers of conduction such as the AVJ (Fig. 2), optical action potentials (OAPs) contain multiple components originating in different layers. The upstroke of individual OAP components can be used to create activation maps and conduction velocity vectors to determine the direction of wave front propagation. As shown in Fig. 3, conduction velocity vectors and fiber orientation vectors are strongly correlated. Therefore, we hypothesized that fiber orientation vectors obtained with OCT and conduction velocity vectors constructed from OAP components recorded from multilayer tissue could be used to identify which layer of tissue was the origin of individual optical signal components.

Bimodal imaging in the AVJ was performed $(n=7)$ to reconstruct the origin of OAP components. Figure 4(a) shows an activation map of retrograde conduction through the AVJ that was induced by pacing the His bundle. Figure 4(b) displays the OCT volume from this preparation. As can be seen in Figs. 4(c)-4(e), components of the activation map can be assigned to different layers of tissue in the OCT volume based on conduction velocity and fiber orientation vectors. In Fig. 4(c), conduction spread from right to left at $20 \mathrm{~cm} / \mathrm{s}$. An example OAP in Fig. 4(c) exhibits two distinct upstrokes. The portion of the activation map shown in Fig. 4(c) was constructed from any OAP that contained the initial upstroke. In the deep tissue layers seen in OCT (700 to $1100 \mu \mathrm{m}$ below the surface), the fibers of the conduction system were oriented approximately parallel to the conduction velocity vectors [Fig. 4(c)].

In Fig. 4(d), conduction propagated very slowly with OAPs showing no distinct upstroke during this period. Instead, the small initial depolarization of the OAP in Fig. 4(d) is consistent with electrotonic activation in this region. The OCT data from this region shows that $400 \mu \mathrm{m}$ below the tissue surface is the interface between the conduction system and the atrial myocardium, where conduction spread transverse to fiber orientation. Finally, the activation map in Fig. 4(e), constructed from OAPs that only contained one upstroke that corresponded in time to the second upstroke seen in Fig. 4(c), demonstrates that activation spread rapidly $(65 \mathrm{~cm} / \mathrm{s})$ in all directions. Optical coherence tomography of the superficial layer of atrial tissue, $50 \mu \mathrm{m}$ below the surface, revealed that fiber orientation in the atrial tissue matched the initial direction of rapid conduction. Accounting for the 3D conduction of activation from the conduction system below the surface to the atrial layer above, conduction spread approximately 3 to $5 \mathrm{~cm} / \mathrm{s}$ between the two layers transversely to fiber orientation. Similar 3D reconstructions of conduction were reproduced in all studied AVJ preparations $(n=7)$.

\subsection{Structural Heterogeneities Contributing to Arrhythmia}

Optical coherence tomography and optical mapping can also be used to investigate structural heterogeneities contributing to arrhythmias. Previous studies have shown that heterogeneities such as disrupted cell-cell coupling and scar tissue can provide the substrate to anchor reentrant arrhythmias, ${ }^{24,25}$ converting paroxysmal arrhythmia to sustained monomorphic tachycardia. Here, we explored the role of microscopic structural heterogeneities in arrhythmia maintenance in the rabbit isolated right ventricle [Fig. 5(a)]. Reentrant arrhythmias were induced in four preparations, and reentry tended to anchor at only one or two locations. These reentry core locations were imaged with OCT. In Fig. 5(a), a preparation with two reentry cores is shown, with a phase map of reentry shown in Fig. 5(b). Surface OCT imaging of each core is shown in Figs. 5(c) and 5(d). The endocardium in Fig. 5(c) is highly trabeculated, and we postulate that this contributed to reentry anchoring at this location.

The reentry core of Fig. 5(d) shows little or no endocardial structural heterogeneity. However, OCT imaging sectioned in the $z^{\prime}$ dimension indicated two distinct myocardial sheets merged in this area. Figures 5(e)-5(g) show OCT images and fiber orientation vectors from superficial to deeper layers: 60, 200 , and $350 \mu \mathrm{m}$ below the surface, respectively. The domi- 

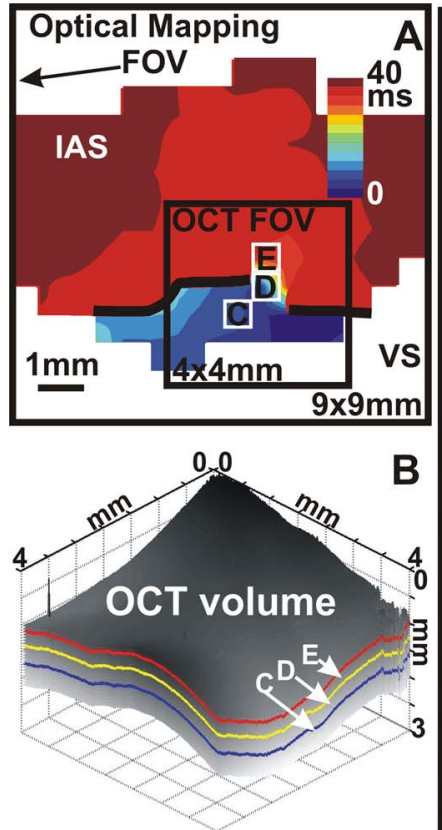

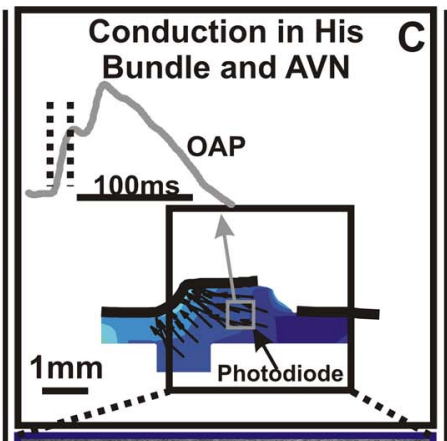

Fiber Orientation in His Bundle and AVN IAS

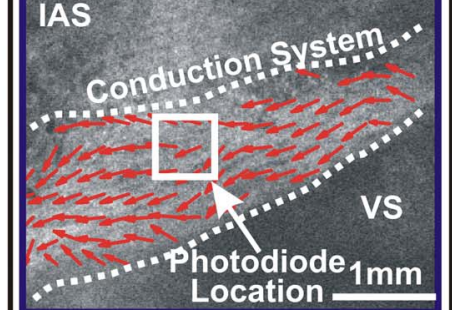

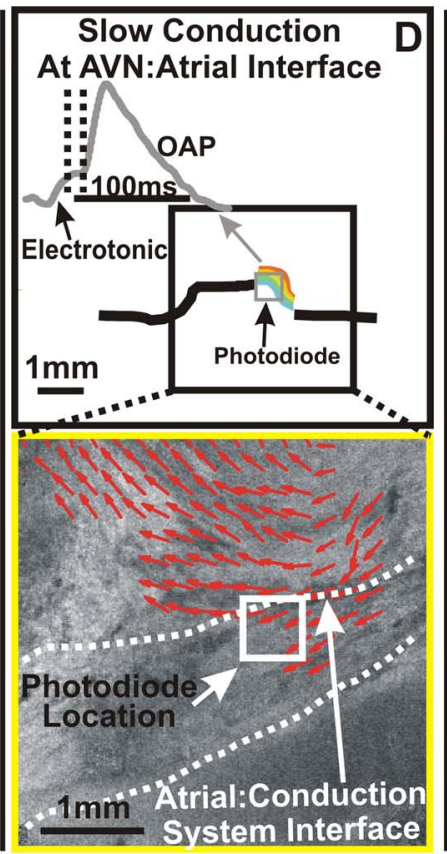

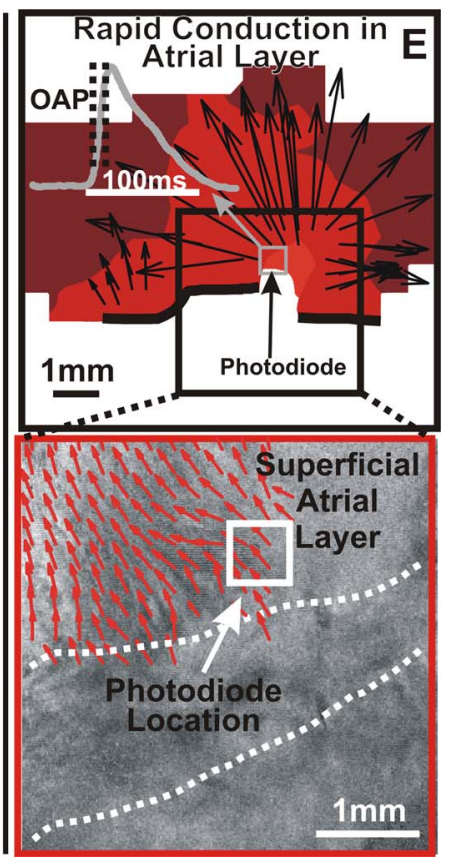

Fig. 4 (a) Activation map of retrograde conduction recorded from the AVJ, where activation began at the lower right, spread to the left, and after a delay spread quickly across the rest of the tissue. Black lines represent lines of conduction block. (b) Volume of OCT data recorded from the same AVJ. The colored lines represent the location of the OCT images shown in panels (c) to (e). (c) Initial 15 ms of the activation map shown in panel (a), with conduction velocity vectors. An OAP recorded from photodiode marked is also shown, with the dotted lines representing the time window corresponding to the $15 \mathrm{~ms}$ of the activation map. The OCT image indicates the location of the conduction system and the fiber orientation that was present in the image. (d) Subsequent $18 \mathrm{~ms}$ of the activation map shown in panel (a) with an OAP recorded from the photodiode marked, and the dotted lines representing the time period shown in the activation map. The OCT image indicates the interface between the conduction system and the atrial myocardium and the fiber orientation in the image. (e) Final $7 \mathrm{~ms}$ of the activation map shown in panel (a), with conduction velocity vectors and OAP recorded from the photodiode marked and the dotted lines representing the time period shown in the activation map. The OCT image indicates the fiber orientation in the superficial atrial myocardium. (Color online only.)
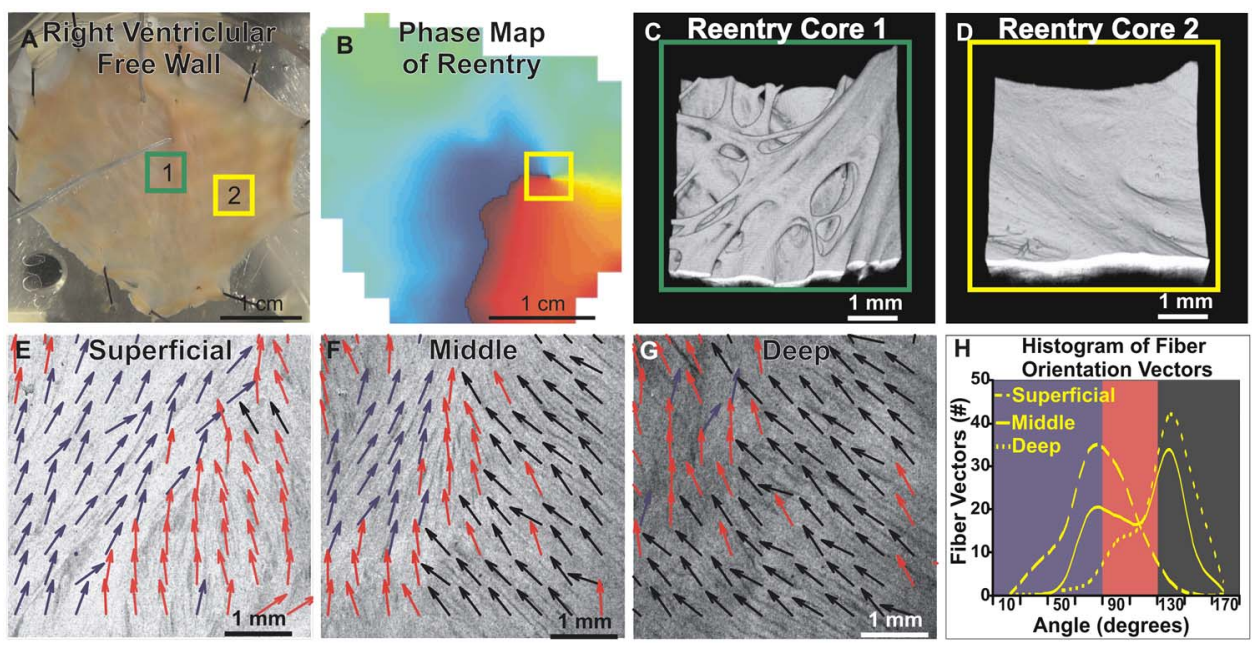

Fig. 5 (a) Picture of rabbit right ventricular free wall preparation. Arrhythmias were anchored in two locations in this preparation marked by the yellow and green boxes as reentry core 1 and reentry core 2. Optical coherence tomography was performed on these regions. (b) Phase map of reentrant arrhythmia, which rotated around reentry core 2 located in the yellow box. (c, d) Surface images of OCT data sets recorded from reentry cores 1 and 2 marked with the the green and yellow locations shown in (a). The complex surface in (c) may have been responsible for anchoring arrhythmias in this location, however the surface of (d) is relatively smooth. (e to g) Progressively deeper OCT images of the data shown in (d) sectioned in $z^{\prime}$ from 60,200 , and $350 \mu \mathrm{m}$ below the surface, respectively, with fiber orientation vectors color coded according to angle. (h) Distributions of the fiber orientation vectors shown in (e to g), which indicate a shift in dominant angle from the superficial layers to the deeper layers in the tissue. Blue, red, and black shading in (h) represent the color coding of the vectors in (e to g). (Color online only.) 

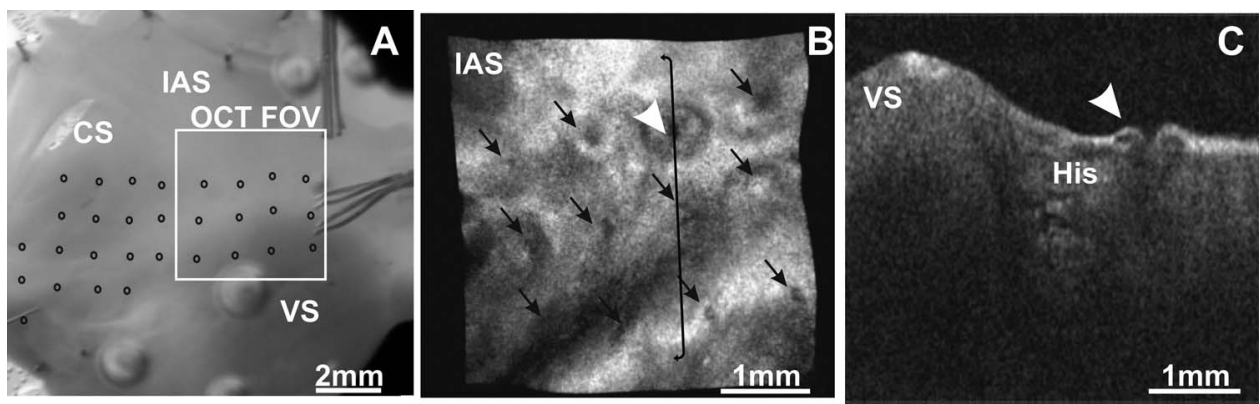

Fig. 6 (a) Preparation of the rabbit AVJ is shown, with different pacing locations marked with circles. The field of view of OCT imaging is indicated. (b) Surface image of OCT data recorded from this preparation. Many areas of tissue damage (marked with black arrows) are present, with the white arrowhead marking the damaged area shown in panel (c). (c) An OCT image perpendicular to the surface shown in (b). The area of tissue damage is surrounded by an area of reduced backscatter. The image shows that the tissue damage did not extend to the His bundle. CS: coronary sinus; IAS: interatrial septum; His: bundle of His; VS: ventricular septum.

nant fiber angle changed from approximately $70 \mathrm{deg}$ in the superficial layer to $130 \mathrm{deg}$ below it [Fig. 5(h)]. Although this area appears quite smooth on the endocardium, the complicated fiber structure revealed with OCT may have played a role in anchoring reentrant arrhythmias in this region.

\subsection{Imaging Tissue Damage}

Functional studies of cardiac tissue often induce damage, either intentionally in the form of ablations, or unintentionally due to pacing. In Fig. 6(a), an AVJ preparation was paced sequentially at each circle from an electrode that roamed in a grid pattern throughout the AVJ. A surface image of the OCT data set [Fig. 6(b)] clearly indicated that pacing at each location produced a small amount of tissue damage. As shown in Fig. 6(c), a section perpendicular to the surface through one of the areas of tissue damage showed tissue swelling and decreased backscattering surrounding the area of injury, indicating that edema surrounded the injury. In addition, it is clear that the area of injury was confined to the superficial atrial layer; the His bundle below the surface was unaffected.

\section{Discussion}

Here, we have illustrated several applications of bimodal biophotonic imaging combining OCT with optical mapping. Using OCT to reconstruct fiber angles from optically mapped preparations provides a precise understanding of the activation patterns recorded and facilitates the interpretation of optical mapping data recorded from preparations with multiple layers of conduction. Optical coherence tomography can also be used to investigate the role of microscopic heterogeneities in arrhythmia maintenance. Finally, we illustrated that OCT is a sensitive technique to investigate tissue damage that results from functional studies. Therefore using OCT to image larger tissue lesions, such as ablations, is certainly possible and could monitor the depth of ablated tissue.

In optical mapping data, the contribution of tissue layers below the surface to the recorded fluorescence is a decaying exponential function, with tissue up to $2 \mathrm{~mm}$ in depth contributing to fluorescence. ${ }^{13}$ Here, we recorded OCT data from the atrial and ventricular myocardium, as well as the cardiac conduction system. We consistently recorded increased depth penetration with OCT in superventricular tissue as compared with the ventricular myocardium. With our system, we could resolve structures 1.5 to $1.8 \mathrm{~mm}$ below the surface of superventricular tissue. However in ventricular tissue, our depth penetration was limited to $\sim 1 \mathrm{~mm}$. Because the depth of penetration of OCT and optical mapping are similar, the two techniques complement each other well with structural and functional data from overlapping tissue volumes.

Histology remains the gold standard for investigating the microscopic structure of biological tissue. However, histological methods are associated with artifacts related to the trauma of sectioning, the plane of section, and tissue processing following sectioning. Here, we introduced a new method of sectioning an OCT volume to create images parallel to the tissue surface, producing images within the myocardium in planes that were physiologically relevant. It is important to note, however, that although this method of sectioning OCT data is valid for smooth surfaces, it is not applicable to trabeculated regions because when there are substantial anatomic variations in the surface, our sectioning method juxtaposes pixels that were not related to each other in the actual tissue.

This form of "virtual histology" can be combined with traditional histology and immunohistochemistry to accurately determine molecular and structural characteristics of particular locations within the intact structure of tissue. When combined with optical mapping, the functional characteristics of such locations can be characterized as well. For example, the data in Fig. 4(d) indicate that slow conduction occurred at the interface of the atrial myocardium and the $\mathrm{AV}$ conduction system, transverse to fiber orientation. This data raises the question of why conduction slowed in this region. Was conduction slow simply because it was transverse to fiber orientation, or did the molecular characteristics of this region play a role? Perhaps when excitation left the densely packed myocardium of the conduction system and entered the loosely packed atrial myocardium surrounding the conduction system, only a few myocytes were depolarized at a time producing what looked like electrotonic activation in the optical signals. After excitation meandered through the loosely packed myocardium, it excited the well-defined superficial atrial myocardium and spread quickly across the atrial septum.

Applying an integrated multimodal imaging with optical mapping, OCT, and immunohistochemistry can enhance our understanding of the mechanisms responsible for slow $\mathrm{AV}$ nodal conduction by pinpointing the precise location of slow 
conduction, determining its intact structure, and then investigating the molecular characteristics of the slowly conducting regions. We have also shown that a similar comprehensive understanding of structural contributions to arrhythmia maintenance, as well as the relationship between structure and function in the sinoatrial node can be investigated using this method. More broadly, this approach can bridge the gap between structural and functional studies in other areas of research, such as neuroscience.

\section{Acknowledgments}

Funding for this project was provided by NIH (R01HL67322), Medtronic, and a Whitaker Foundation Graduate Fellowship.

\section{References}

1. D. Huang, E. A. Swanson, C. P. Lin, J. S. Schuman, W. G. Stinson, W. Chang, M. R. Hee, T. Flotte, K. Gregory, C. A. Puliafito et al., “Optical coherence tomography," Science 254, 1178-1181 (1991).

2. J. G. Fujimoto, "Optical coherence tomography for ultrahigh resolution in vivo imaging," Nat. Biotechnol. 21, 1361-1367 (2003).

3. A. G. Podoleanu, "Optical coherence tomography," Br. J. Radiol. 78, 976-988 (2005)

4. M. Jenkins, R. S. Wade, Y. Cheng, A. M. Rollins, and I. R. Efimov, "Optical coherence tomography imaging of the purkinje network," $J$. Cardiovasc. Electrophysiol. 16, 559-560 (2005).

5. B. E. Bouma and G. J. Tearney, Handbook of Optical Coherence Tomography, Marcel Dekker Inc, New York, N.Y. (2002).

6. S. J. Bakri, A. D. Singh, C. Y. Lowder, M. R. Chalita, Y. Li, J. A Izatt, A. M. Rollins, and D. Huang, "Imaging of iris lesions with high-speed optical coherence tomography," Ophthalmic Surg. Lasers 38, 27-34 (2007).

7. Y. Chen, P. M. Andrews, A. D. Aguirre, J. M. Schmitt, and J. G. Fujimoto, "High-resolution three-dimensional optical coherence tomography imaging of kidney microanatomy ex vivo," J. Biomed. Opt. 12, 034008 (2007).

8. H. Yabushita, B. E. Bouma, S. L. Houser, H. T. Aretz, I. K. Jang, K. H. Schlendorf, C. R. Kauffman, M. Shishkov, D. H. Kang, E. F. Halpern, and G. J. Tearney, "Characterization of human atherosclerosis by optical coherence tomography," Circulation 106, 1640-1645 (2002).

9. M. W. Jenkins, O. Q. Chughtai, A. N. Basavanhally, M. Watanabe, and A. M. Rollins, "In vivo gated 4D imaging of the embryonic heart using optical coherence tomography," J. Biomed. Opt. 12, 030505 (2007).

10. B. A. Filas, I. R. Efimov, and L. A. Taber, "Optical coherence tomography as a tool for measuring morphogenetic deformation of the looping heart," Anat. Rec. 290, 1057-1068 (2007).
11. M. Gupta, A. M. Rollins, J. A. Izatt, and I. R. Efimov, "Imaging of the atrioventricular node using optical coherence tomography," $J$. Cardiovasc. Electrophysiol. 13, 95 (2002).

12. I. R. Efimov, V. P. Nikolski, and G. Salama, "Optical imaging of the heart," Circ. Res. 95, 21-33 (2004).

13. W. T. Baxter, S. F. Mironov, A. V. Zaitsev, J. Jalife, and A. M. Pertsov, "Visualizing excitation waves inside cardiac muscle using transillumination," Biophys. J. 80, 516-530 (2001).

14. V. G. Fast, "Simultaneous optical imaging of membrane potential and intracellular calcium," J. Electrocardiol. 38, 107-112 (2005).

15. L. Li, V. P. Nikolski, D. W. Wallick, I. R. Efimov, and Y. Cheng, "Mechanisms of enhanced shock-induced arrhythmogenesis in the rabbit heart with healed myocardial infarction," Am. J. Physiol. Heart Circ. Physiol. 289, H1054-H1068 (2005).

16. C. M. Ripplinger, V. I. Krinsky, V. P. Nikolski, and I. R. Efimov, "Mechanisms of unpinning and termination of ventricular tachycardia," Am. J. Physiol. Heart Circ. Physiol. 291, H184-H192 (2006).

17. I. R. Efimov, G. J. Fahy, Y. Cheng, D. R. Van Wagoner, P. J. Tchou, and T. N. Mazgalev, "High-resolution fluorescent imaging does not reveal a distinct atrioventricular nodal anterior input channel (fast pathway) in the rabbit heart during sinus rhythm," J. Cardiovasc. Electrophysiol. 8, 295-306 (1997).

18. P. V. Bayly, B. H. KenKnight, J. M. Rogers, R. E. Hillsley, R. E. Ideker, and W. M. Smith, "Estimation of conduction velocity vector fields from epicardial mapping data," IEEE Trans. Biomed. Eng. 45 , 563-571 (1998).

19. Z. Hu and A. M. Rollins, "Quasi-telecentric optical design of a microscope-compatible OCT scanner," Opt. Express 13, 6407-6415 (2005).

20. C. P. Fleming, C. M. Ripplinger, B. Webb, I. R. Efimov, and A. M. Rollins, "Quantification of cardiac fiber orientation using 3D optical coherence tomography," J. Biomed. Opt. 13, 030505 (2008).

21. W. J. Karlon, J. W. Covell, A. D. McCulloch, J. J. Hunter, and J. H. Omens, "Automated measurement of myofiber disarray in transgenic mice with ventricular expression of ras," Anat. Rec. 252, 612-625 (1998).

22. M. Valderrabano, "Influence of anisotropic conduction properties in the propagation of the cardiac action potential," Prog. Biophys. Mol. Biol. 94, 144-168 (2007).

23. V. V. Fedorov, W. J. Hucker, H. Dobrzynski, L. V. Rosenshtraukh, and I. R. Efimov, "Postganglionic nerve stimulation induces temporal inhibition of excitability in rabbit sinoatrial node," Am. J. Physiol. Heart Circ. Physiol. 291, H612-H623 (2006).

24. N. S. Peters, J. Coromilas, N. J. Severs, and A. L. Wit, "Disturbed Connexin43 gap junction distribution correlates with the location of reentrant circuits in the epicardial border zone of healing canine infarcts that cause ventricular tachycardia," Circulation 95, 988-996 (1997).

25. A. L. Wit, S. M. Dillon, J. Coromilas, A. E. Saltman, and B. Waldecker, "Anisotropic reentry in the epicardial border zone of myocardial infarcts," Ann. N.Y. Acad. Sci. 591, 86-108 (1990). 